\title{
Association rules mining applied in the animal movement exploratory analysis
}

\author{
S. G. Fontes ${ }^{1}$, P. L. P. Côrrea ${ }^{1}$, S. L. Stanzani², R. G. Morato ${ }^{3}$ \\ 1 Escola Politécnica Universidade de São Paulo (USP), Brasil \\ suelane@usp.br, pedro. correa@usp.br \\ 2 Centro de Computação Científica - Universidade Estadual Paulista Júlio de Mesquita Filho (UNESP), Brasil \\ silvio@ncc.unesp.br \\ ${ }^{3}$ Centro Nacional de Pesquisa e Conservação de Mamíferos Carnívoros (CENAP)/ICMBIO, Brasil \\ ronaldo.morato@icmbio.gov.br
}

\begin{abstract}
The animal movement analysis determines the animal behavior, which is the basis for understanding the interaction between species and the environment and to guide actions of preservation and conservation. The challenge is how to explore this movement data, getting indications about how the animal behaves over time and space. In this sense, a framework to animal movement exploratory analysis is presented, that combines algorithms for spatiotemporal data analysis and association rules mining, as a first step to answer questions related to animal behavior. We performed the framework's evaluation in the exploratory analysis of monitored monkeys (Cebus capucinus) in the Panamá.
\end{abstract}

Categories and Subject Descriptors: H.2.8 [Database Management]: Database Applications - Data Mining

Keywords: animal behavior, animal movement, association rules mining, data science, spatiotemporal data analysis

\section{INTRODUÇÃO}

O comportamento animal é a caracterização da forma como o animal interage com o ambiente [Snowdon 1999] e analisar essa interação pode fornecer subsídios para identificar como as ações humanas estão prejudicando o meio ambiente, o que é de suma importância, considerando que a sociedade tem vivenciado grandes mudanças ambientais e catástrofes naturais causadas pelas ações humanas. Um meio de identificar o comportamento do animal é a análise de sua movimentação, considerando que esses dados espaço-temporais descrevem a trajetória do animal, sendo formados por pontos distribuídos no tempo e espaço. Ao longo dessas trajetórias ocorrem os fatores ambientais, tais como, chuvas, queimadas e a presença de outros animais, que podem ou não influenciar no comportamento do animal.

Com os avanços tecnológicos, o uso de sensores e global positioning systems (GPS) para o rastreamento de animais foi intensificado, ampliando a coleta e a disponibilização de dados espaço-temporais, relativos à movimentação animal, e a capacidade de acompanhamento, estudo e gerenciamento desses movimentos. Algumas instituições que fazem o rastreamento de animais são: o Instituto Max Planck de Ornitologia [Planck 2019]) e o Centro Nacional de Pesquisa e Conservação de Mamíferos Carnívoros [CENAP 2019]. Observa-se, portanto, que os dados espaço-temporais de movimentação animal estão disponíveis para pesquisa e com base na distribuição desses dados pode-se identificar o comportamento animal. No entanto, o comportamento corresponde à caracterização da forma como o animal interage com o ambiente exigindo que sejam realizadas análises diversas para que se obtenha indicativos sobre seu comportamento. Diante disso, o desafio é como realizar a análise exploratória dos dados de movimentação animal para obter indicativos sobre o comportamento animal ao longo do tempo e espaço.

Copyright(C)2019 Permission to copy without fee all or part of the material printed in KDMiLe is granted provided that the copies are not made or distributed for commercial advantage, and that notice is given that copying is by permission of the Sociedade Brasileira de Computação. 
Desta forma, neste artigo é apresentado o framework AniMoveMineR, que tem como objetivo permitir a análise exploratória do movimento animal, ao longo do tempo e espaço, por meio da combinação de algoritmos de análise de dados espaço-temporais e de mineração de regras de associação. Com a análise exploratória do movimento animal, que abrange a classificação do comportamento em estados e a identificação de quando, onde e por quanto tempo estes estados de comportamento ocorreram e da correlação entre os animais e os fatores ambientais, pretende-se oferecer aos pesquisadores e órgãos públicos um meio de obter conhecimento sobre o comportamento animal que oriente as ações de preservação e conservação do meio ambiente. Desta forma, a contribuição deste artigo é apresentar o framework proposto AniMoveMineR e sua aplicação no campo da biodiversidade por meio de um estudo de caso, utilizando dados de movimentação de macacos (cebus capucinus) da região de Barro Colorado Island no Panamá.

\section{REFERENCIAL TEÓRICO}

O movimento animal é a variação da posição espacial de um objeto em relação a um referencial no período de tempo, gerando um caminho representado por uma série de pontos ordenados no tempo, a trajetória. A análise de trajetórias [Demsar et al. 2015] pode fornecer informações sobre por que, como, quando e onde os animais se movem e sua relação com o ambiente [Phan 2013]. O movimento animal possui características, tais como velocidade, tempo de residência, direção e distância percorrida ou a frequência de ocorrência que permitem identificar padrões de comportamento animal. O comportamento animal descreve como os animais interagem com o ambiente [Snowdon 1999] e divide-se em: estados, que ocorrem por período prolongado; e eventos, que são contados ao longo do tempo.

[Zhang et al. 2015], analisa os estados do comportamento, combinando o método Behavioral Change Point Analysis (BCPA) [Gurarie et al. 2009], que segmenta as trajetórias baseado na velocidade e direção do movimento, e a técnica de clusterização k-means para classificar as trajetórias dos animais em estados. Os métodos de segmentação particionam as trajetórias com base nas características do movimento, determinando os pontos que indicam o local e o momento da transição do comportamento [Edelhoff et al. 2016]. O método Residence in Space and Time (RST) [Torres et al. 2017] segmenta trajetórias baseado no tempo de residência e distância e o AdehabitatLT [Calenge and Royer 2015] fornece classes e métodos para manipular, analisar e segmentar trajetórias animais.

Com base nas trajetórias dos animais identifica-se o home range animal, que é a área percorrida pelo animal na busca de alimento, acasalamento e cuidado parental [Burt 1943]. O AdehabitatHR [Calenge 2006] fornece classes e métodos para analisar e identificar o home range. O movimento animal ocorre em uma determinada área e pode ser influenciado por outros objetos que estão no mesmo ambiente, podendo ocorrer relações, tais como, de: coocorrência, representa subconjuntos de eventos que ocorrem juntos no espaço e no tempo; correlação [Mari and Kotz 2001], relação de dependência estatística entre duas ou mais variáveis ocorrendo no mesmo espaço e tempo.

Em Ciência de Dados, a mineração de dados é parte da etapa de análise dos dados e compreende o conjunto de estratégias, ferramentas e algoritmos que permitem a exploração dos dados e a extração de padrões, sendo aplicada em diferentes áreas como prevenção de fogo em florestas [Cheng and Wang 2008], descoberta de rebanhos por clusterização [Jacob and Idicula 2012] e associação entre produtos de cestas de compras por meio de regras de associação [Brin et al. 1997].

\section{ANIMOVEMINER - FRAMEWORK PROPOSTO}

O framework AniMoveMineR permite a análise exploratória do movimento animal ao longo do tempo e espaço, utilizando uma combinação de algoritmos de análise de dados espaço-temporais e de mineração de regras de associação, com o objetivo de obter indicativos sobre o comportamento animal. O AniMoveMineR se baseia no processo da Ciência dos Dados [Dekhtyar 2016] e considera que no processo de análise deve-se formular questões a serem respondidas, os dados devem ser recuperados e 
preparados e a análise dos dados deve ser realizada, fornecendo resultados para serem avaliados pelos pesquisadores.

O framework recebe dois datasets: um com a movimentação animal, caracterizada por pontos de localização (latitude/longitude), tempo (data/hora) do movimento e nome do animal; e o outro com os fatores ambientais, contendo o nome do fator, posição (latitude/longitude), tempo (data/hora) de ocorrência e o status que identifica o fator, por exemplo, status da temperatura (alta/baixa).

$\mathrm{Na}$ etapa de análise dos dados, conforme Fig. 1, é realizada a análise exploratória do movimento animal sendo dividida em três etapas: (A) Identificar os estados do comportamento animal; (B) Identificar os fatores ambientais vizinhos da ocorrência do comportamento animal; (C) Identificar a correlação entre os animais e os fatores ambientais.

Na etapa A, identificar os estados do comportamento animal, é realizada a classificação do comportamento em estados por meio da segmentação das trajetórias do animal, usando o método RST [Torres et al. 2017], que cria um círculo ao redor de cada ponto do movimento animal, de acordo com o Thresold distance (TD) que é o tamanho do raio em metros, sendo calculada a distância percorrida (RD) e o tempo gasto (RT) entre pontos consecutivos dentro do círculo. Os valores de RD e RT são normalizados, obtendo-se os valores entre 0 e 1 , e subtraídos (RT - RD), gerando o valor de resíduo entre -1 e 1 . Com base no valor de resíduo, cada ponto da trajetória é classificado como: descanso (resíduo < 0), um tempo alto na área, mas uma curta distância percorrida; trânsito (resíduo $=0$ ), baixo tempo e distância na área; forrageamento (resíduo > 0), alto tempo e distância na área.

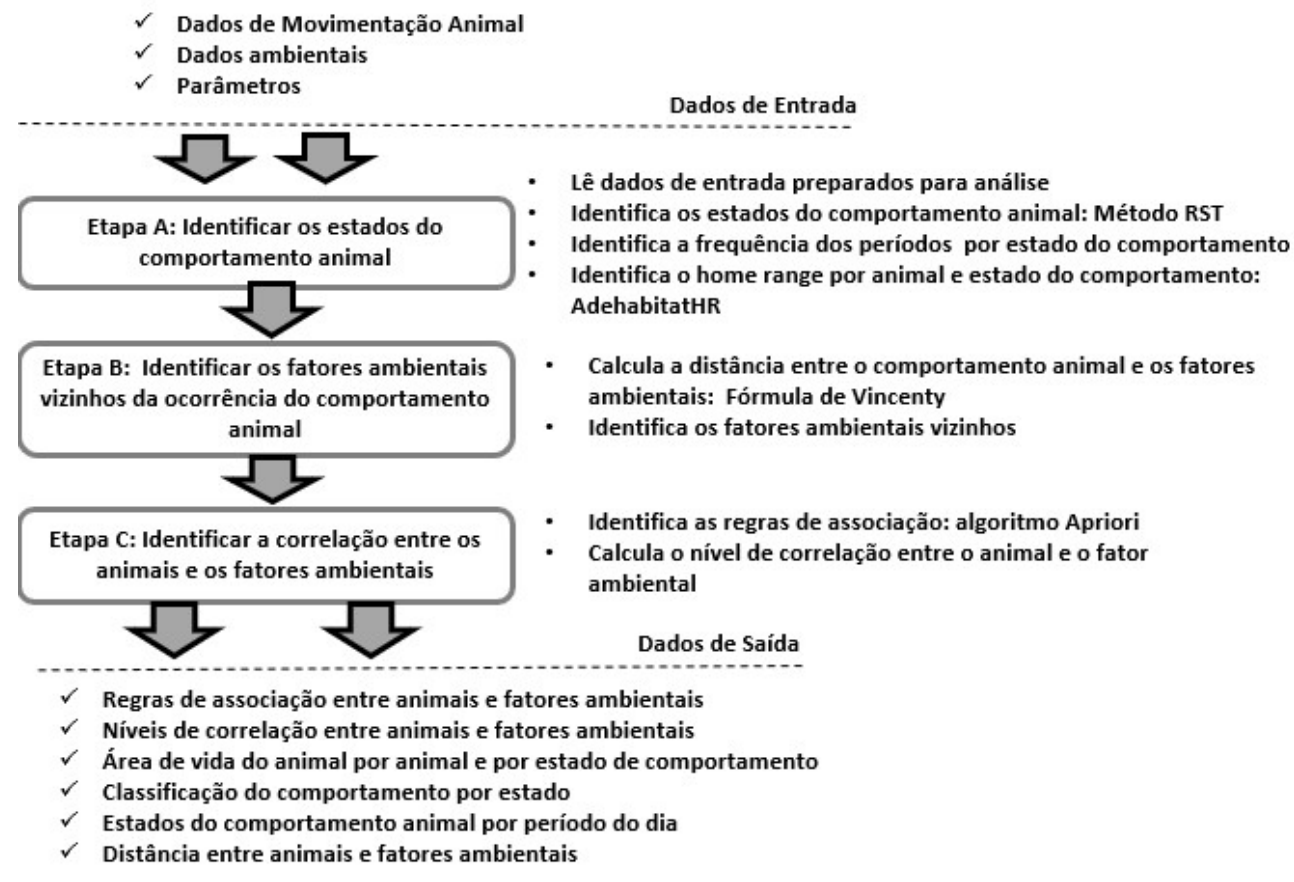

Fig. 1. Etapas da Análise de Dados AniMoveMineR

Após a classificação dos estados, são identificados os períodos (hora início/término) de ocorrência dos estados por animal/dia, o home range por animal e por animal e estado de comportamento e a frequência da ocorrência dos estados por período do dia. A duração dos períodos é usada para identificar e remover os outliers dos períodos, usando o pacote outliers [Komsta 2011]. Os outliers são registros de duração do estado de comportamento que diferem fortemente dos outros e podem causar anomalias nos resultados das análises. Para identificar os períodos frequentes, é calculada a frequência de ocorrência dos estados por intervalo entre horas (início/fim). 
Para identificar a área de vida dos animais é utilizada a função kernelUD [Calenge 2006], que estima a densidade de probabilidade de um animal ser encontrado em um ponto de acordo com suas coordenadas geográficas, definindo a área de vida como a área mínima em que um animal tem alguma probabilidade específica de localização.

Na etapa B, identificar os fatores ambientais vizinhos da ocorrência do comportamento animal, os dados de movimentação animal e fatores ambientais são analisados para identificar os fatores presentes ao longo dos pontos de movimento. Há duas situações: na primeira situação, os fatores possuem informações sobre espaço (latitude/longitude) e tempo, então as datas são sincronizadas e a hora é acrescida de uma margem, em minutos (TI), antes e depois da hora de início e fim da ocorrência do fator e a distância é calculada usando a fórmula de Vincenty [Vincenty 1975], que cria um círculo em torno de cada ponto do movimento com um raio de distância em metros (TD); na segunda situação, os fatores estão na mesma área do movimento animal, mas há somente informações sobre tempo, então a data do movimento é sincronizada com a data do fator, considerando acréscimo de minutos (TI), antes e depois.

$\mathrm{Na}$ etapa C, identificar a correlação entre o comportamento animal e os fatores ambientais, são definidas as variáveis a serem analisadas e o algoritmo Apriori [Hahsler et al. 2005] é aplicado para identificar a correlação entre as variáveis, por meio das regras de associação que encontram elementos que implicam na presença de outros elementos no mesmo dataset, indicando a freqüência de coocorrência desses elementos. Os dados de entrada para o Apriori são: ao menos duas variáveis, por exemplo, se o objetivo é identificar a correlação entre o estado de comportamento animal e o fator ambiental focos de calor, então, as variáveis de estado de comportamento e status do fator são recuperadas.

O framework realiza as análises com MinSup e MinConf, usados para identificar as regras de associação relevantes, definidos como: Baixo (0.01), igual a 1\%, recupera as regras com baixa frequência de coocorrência para as variáveis; Intermediário (0.50), igual a 50\%, recupera as regras com intermediário valor de frequência de coocorrência das variáveis; Alta (0.9), igual a 90\%, recupera as regras com alta frequência de coocorrência das variáveis. Quanto menor o valor de MinSup e MinConf, mais relacionamentos entre variáveis são recuperados, no entanto, até mesmo raros encontros entre animais serão mostrados. Quanto maior o valor, maior o filtro das relações, mostrando apenas as com maior probabilidade de ocorrência.

O Apriori identifica as regras de associação entre as variáveis A e B, considerando a frequência com que ocorrem em conjunto e eliminando as regras que estão abaixo dos limites de MinSup e MinConf. Como resultado, são obtidas as regras de associação e as medidas que identificam a correlação e dependência entre as variáveis analisadas [Manimaran and Velmurugan 2015]. As medidas obtidas indicam a frequência de coocorrência (Sup) das duas variáveis (A e B); a frequência de ocorrência somente da variável A (SupA) e da B (SupB); a frequência esperada (SupEsp) de coocorrência das variáveis A e B; e a confiança (Conf), que indica a probabilidade de B ocorrer em função da ocorrência da variável A. Outra medida obtida é o coeficiente de correlação (Phi) que indica a força da relação entre A e B, classificando o nível de correlação [Taylor 1990] como: correlação negativa perfeita (-1); forte correlação negativa (-1.0 a -0.68); moderada correlação negativa (-0.67 a -0.36$)$; fraca ou nenhuma correlação (-0.35 a 0.35$)$; moderada correlação positiva ( 0.36 a 0.67$)$; forte correlação positiva (0.68 a 1.0); sem correlação (0); correlação positiva perfeita (1).

\section{ESTUDO DE CASO}

Nesta Seção é apresentado o uso do framework AniMoveMineR na análise exploratória de um dataset contendo a movimentação de 12 macacos (Cebus Capucinus), utilizado em [Li et al. 2013], referentes aos anos de 2004 e 2005 na região de Barro Colorado Island - Panamá. Esses macacos são divididos em 6 grupos (pares de animais), conforme Tabela I. A hipótese considerada neste estudo é que explorando os dados de movimentação dos macacos pode-se obter indicativos sobre quais animais pertencem ao mesmo grupo. A análise exploratória dos dados foi executada, considerando as três etapas que compõem este passo: (A) Identificar os estados de comportamento animal; (B) Identificar 


\begin{tabular}{ccccccc} 
& Table I. & \multicolumn{6}{c}{ Grupos de macacos $($ cebus capucinus $)$} \\
\hline Grupo & TB & BLT & Top & BL12 & FC & Conrad \\
\hline ID Animal & $51-87$ & $52-53$ & $54-55$ & $57-58$ & $83-84$ & $85-86$ \\
\hline
\end{tabular}

os fatores ambientais vizinhos da ocorrência do comportamento animal; (C) Identificar a correlação entre os animais e os fatores ambientais. Na etapa A, foi realizada a análise exploratória dos dados de movimentação dos macacos com o foco nos estados do comportamento desses animais, obtendo-se a classificação do comportamento em estados (descanso, trânsito e forrageamento), os períodos de ocorrência dos estados (dia/noite), conforme Tabela II, bem como a duração (hora início e fim) da ocorrência do estado e a área ocupada pelo animal ao longo do tempo. Com esses indicativos pode-se responder questões sobre o comportamento animal, tais como: Quais os horários que ocorrem os estados de comportamento para cada animal, mês e ano? Qual o percentual de ocorrência de cada estado por período do dia (dia/noite) para cada animal, mês e ano? Qual a área de vida do animal e quais animais compartilharam o mesmo espaço?

A Tabela II, mostra que o macaco 51 forrageou das 11:49:44 às 15:38:14 em novembro/2004. Com

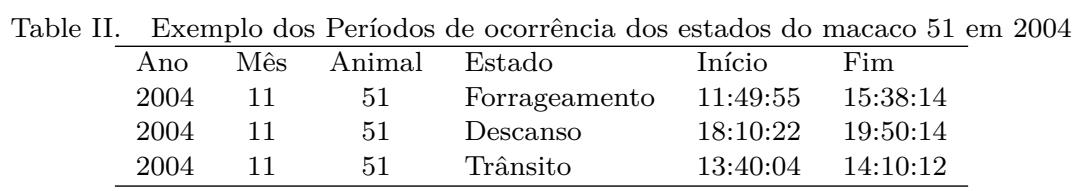

base nos períodos de ocorrência dos estados de comportamento pode-se identificar como um animal se comporta em relação ao dia e noite. A Tabela III, mostra o exemplo do macaco 51 que foi identificado forrageando à noite 29 vezes em janeiro de 2005. Os períodos foram classificados como D (dia), N (noite) e DN (Dia/Noite). O período do D (dia) abrange o horário das 6h da manhã às 17:59:59h e das Oh às 05:59:59h. O período da N (Noite) abrange das 18h às 23:59:59h. O período de DN (Dia/Noite) compreende os intervalos entre horas, que começam durante o dia e terminam a noite.

$\mathrm{Na}$ etapa B, foram identificados os fatores ambientais vizinhos da ocorrência do comportamento ani-

Table III. Estados do comportamento do animal 51 por período do dia (dia/noite) - 2005

\begin{tabular}{lcclll}
\hline Ano & Mês & Animal & Estado & Período & total \\
2005 & 1 & 51 & Forrageamento & N & 29 \\
2005 & 1 & 51 & Forrageamento & DN & 13 \\
2005 & 1 & 51 & Forrageamento & D & 8 \\
2005 & 1 & 51 & Descanso & N & 12 \\
\hline
\end{tabular}

mal usando a distância entre os animais e o fator ambiental. Neste caso, o fator ambiental é a presença de outros animais. Desta forma, todas as ocorrências de movimento foram analisadas sincronizando as datas de movimentação e calculando as distâncias entre os pontos (latitude/longitude), utilizando a fórmula de Vincenty para um raio (TD) menor ou igual a 100 metros.

$\mathrm{Na}$ etapa C, a análise dos dados foi aplicada para identificar a correlação entre os animais, usando a mineração por regras de associação com o algoritmo Apriori. Como o Apriori utiliza os parâmetros MinSup e MinConf para determinar quais regras são relevantes o framework realizou as análises considerando os valores: Baixo (0.01), referente a 1\% para recuperar até mesmo as regras de associação com baixa frequência de coocorrência para as variáveis; Intermediário (0.50), referente a $50 \%$ para recuperar as regras de associação com um valor intermediário de frequência de coocorrência das variáveis; Alta (0.9), referente a 90\% para recuperar as regras de associação que apresentam alta frequência de coocorrência das variáveis.

Com a análise exploratória foi identificado que o dataset continha dados da movimentação dos macacos (Cebus Capucinus) de dois anos: 2004, meses de Novembro e Dezembro, e 2005, os meses de janeiro, 
fevereiro, março e abril. Para Novembro/2004, a mineração por regras de associação indicou: Forte correlação entre os pares de macacos: 52/53 e 54/55; Moderada correlação entre os macacos: 57/58; Fraca ou nenhuma correlação entre os pares de macacos: 53/51, 53/57 e 55/58. Para dezembro/2004, os animais 52/53 e 54/55 mantiveram a indicação de alta correlação. Na Fig. 2(A), é mostrada a variação dos níveis de correlação entre os animais, indicando maior probabilidade de correlação quando os valores estão mais próximos de $100 \%$ e menor probabilidade quando estão mais próximos de $0 \%$. Os números mostrados em cada quadrado referem-se ao \% de correlação entre cada par de macacos. Observa-se que os pares de animais 52/53 e 54/55 foram indicados em ambos os meses com alto nível

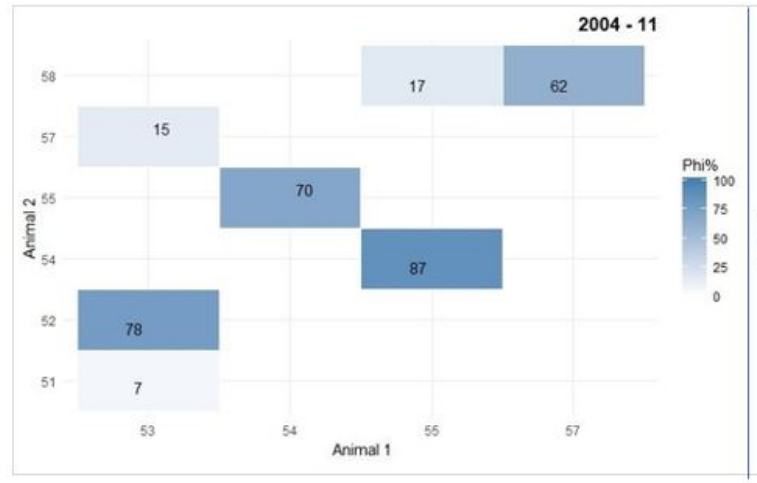

(A)

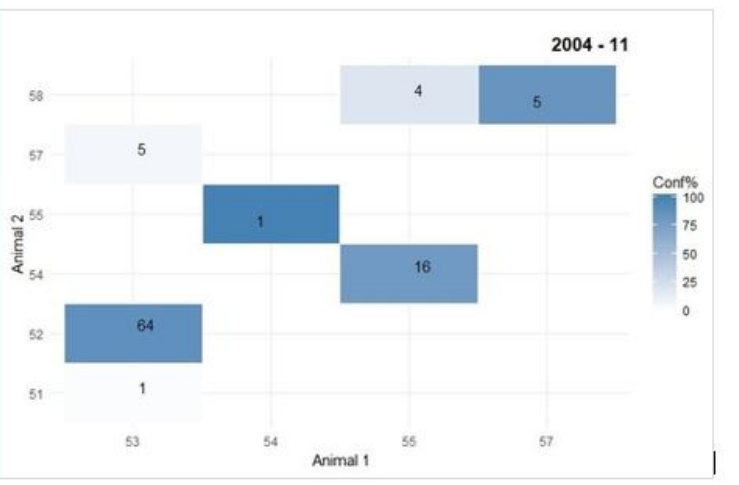

(B)

Fig. 2. Exemplo do gráfico de (A) Nível de correlação, (B) Suporte e Confiança para os dados de Novembro/2004

de correlação. Por meio das regras de associação, também, foram obtidas as medidas, conforme a Fig.2(B), de: suporte, que indica a frequência de coocorrência dos pares de animais, e a confiança, que indica a probabilidade do Animal 1 ocorrer em função da ocorrência do Animal 2. Por exemplo, os macacos 53 e 52, possuem indicativo de forte correlação, baseado no valor de Phi, e analisando o suporte e confiança para essa relação observa-se que foram registradas 64 ocorrências juntos (Suporte), valor mostrado no quadro, e a probabilidade do macaco 53 ocorrer quando o macaco 52 ocorre é igual a $88 \%$ (Confiança), indicado pela intensidade da cor do quadrado na Fig.2(B). Para os macacos 54 e 55 , o valor de suporte é 16 , e a confiança é de $80 \%$. Os dados não contêm os pares de animais 51/87, $83 / 84$ e $85 / 86$.

A análise exploratória dos dados para 2005 indicou que os animais 52/53, 54/55 e 58/57, que apresentaram perfeita ou forte correlação para o ano de 2004, mantiveram esse nível de correlação em 2005, enquanto os animais 51/87 e 85/86 que não constavam nos resultados das análises em 2004, passaram a ser indicados com forte correlação, Fig.3, em Março e Abril de 2005. Comparando os grupos de animais, identificados por [Li et al. 2013] conforme Tabela I, com os pares de macacos correlacionados indicados pelas regras de associação, observa-se que os animais com forte, perfeita ou moderada correlação são animais que pertencem aos grupos, como os animais 52/53, 54/55, 57/58, 51/87 e 85/86. A relação entre esses animais pode ser observada, também, por meio da análise da sobreposição de área de vida (home range) entre os animais [Kernohan et al. 1978], conforme a Fig.4, mostra a proporção de sobreposição da área de vida de um animal em relação aos outros. Quanto mais próximo o valor de 1 maior a sobreposição, desta forma, observa-se que em 2004 os animais 52/53 apresentaram valor 1 , os animais $54 / 55$ o valor é igual a 0.884 e para os animais $58 / 57$ é igual a 0.772 .

Outra forma de verificar a sobreposição é por meio de mapas que mostram a área de vida por animal, mês e ano ou por animal, mês, ano e estado. Na Fig. 5, por exemplo, observa-se que a área onde os animais 54 e 55 forragearam, descansaram e transitaram apresenta sobreposição indicando que esses animais ocuparam a mesma área ao longo do mês de novembro de 2004. Os animais 53/57 


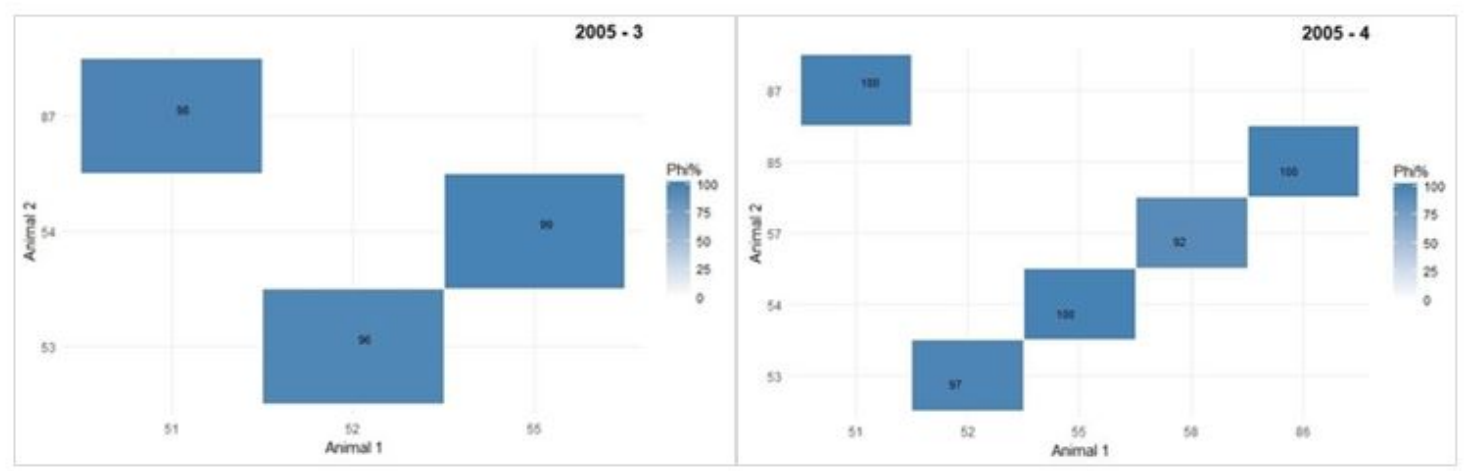

Fig. 3. Nível de Correlação entre macacos em Março e Abril de 2005

51

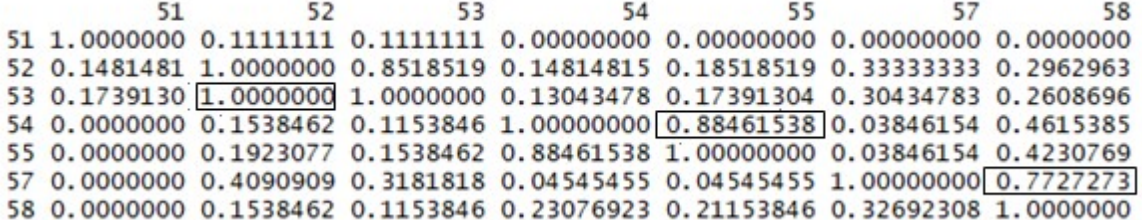

Fig. 4. Proporção de sobreposição da área de vida dos macacos em 2004
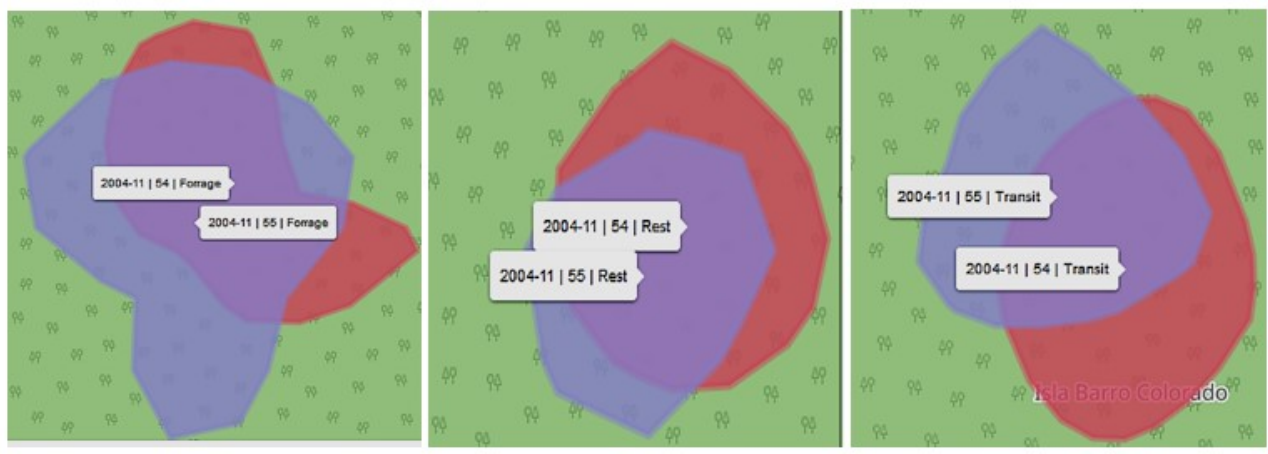

Fig. 5. Área de vida dos macacos 54 e 55 em 2004

apresentaram indicativo de fraca ou nenhuma correlação para novembro/2004 e, observando o home range desses animais verifica-se que não houve sobreposição de área.

\section{CONSIDERAÇÕES FINAIS}

Com o estudo de caso observa-se que o framework AniMoveMineR é o primeiro passo para se obter indicativos sobre o comportamento animal e sobre grupos de animais a partir da exploração dos dados de movimentação dos animais. Com o resultado da análise exploratória dos dados espaço-temporais do movimento dos macacos cebus capucinus usando o framework AniMoveMineR, pode-se: classificar o comportamento dos macacos em estados; identificar a proximidade entre os macacos; identificar os grupos de macacos com base nos níveis de correlação, obtidos com as regras de associação; identificar a área de vida por macaco e estado de comportamento ao longo do tempo (mês/ano). O framework ainda permite identificar a ausência de dados de rastreamento do movimento dos animais em determinados períodos. Os algoritmos utilizados e os dados, gráficos e figuras obtidos com o estudo estão disponíveis 
no repositório GitHub: https://github.com/suelanegarcia/AniMoveMineR.

\section{ACKNOWLEDGMENT}

Este estudo foi financiado em parte pela Coordenação de Aperfeiçoamento de Pessoal de Nível Superior (CAPES) - Código Financeiro 001.

\section{REFERENCES}

Brin, S., Motwani, R., Ullman, J. D., And Tsur, S. Dynamic itemset counting and implication rules for market basket data. In Proceedings of the 1997 ACM SIGMOD International Conference on Management of Data. ACM, New York, NY, USA, pp. 255-264, 1997.

Burt, W. H. Territoriality and home range concepts as applied to mammals. Journal of Mammalogy 24 (3): 346-352, 1943.

Calenge, C. The package adehabitat for the R software: A tool for the analysis of space and habitat use by animals. Ecological Modelling 197 (3): 516-519, 2006.

Calenge, C. And Royer, C. F. S. D. A. M. adehabitatLT: Analysis of Animal Movements. https://cran.rproject.org/web/packages/adehabitatLT/index.html, 2015.

CENAP. Centro nacional de pesquisa e conservação de mamíferos carnívoros. http://www.icmbio.gov.br/cenap, 2019.

Cheng, T. And WAng, J. Integrated Spatio-temporal Data Mining for Forest Fire Prediction. Transactions in GIS 12 (5): 591-611, 2008.

DeкhтүaR, A. Lecture notes on data science - data 301. http://users.csc.calpoly.edu/ dekhtyar/DATA301Spring2016/lectures/lec03.301.pdf., 2016.

Demsar, U., Buchin, K., Cagnacci, F., Safi, K., Speckmann, B., Van de Weghe, N., Weiskopf, D., and Weibel, R. Analysis and visualisation of movement: an interdisciplinary review. Movement Ecology 3 (1): $5,2015$.

Edelhoff, H., Signer, J., And Balkenhol, N. Path segmentation for beginners: an overview of current methods for detecting changes in animal movement patterns. Movement Ecology vol. 4, pp. 21, 2016.

Gurarie, E., Andrews, R. D., And Laidre, K. L. A novel method for identifying behavioural changes in animal movement data. Ecology Letters 12 (5): 395-408, 2009.

Hahsler, M., Gruen, B., And Hornik, K. arules A Computational Environment for Mining Association Rules and Frequent Item Sets. Journal of Statistical Software 14 (15): 1-25, 2005.

JACOB, G. AND IDICUlA, S. Detection of flock movement in spatio-temporal database using clustering techniques - An experience. In 2012 International Conference on Data Science Engineering (ICDSE). IEEE Xplore, Kochi, India, pp. 69-74, 2012.

Kernohan, B. J., Gitzen, R. A., And Millspaugh, J. J. Chapter 5 - Analysis of Animal Space Use and Movements. In J. J. Millspaugh and J. M. Marzluff (Eds.), Analysis of Animal Space Use and Movements. Academic Press, San Diego, pp. 125-166, 1978.

Komsta, L. outliers: Tests for outliers. https://CRAN.R-project.org/package=outliers, 2011.

Li, Z., Ding, B., Wu, F., Lei, T. K. H., Kays, R., And Crofoot, M. C. Attraction and Avoidance Detection from Movements. Proc. VLDB Endow. 7 (3): 157-168, 2013.

Manimaran, J. And Velmurugan, T. Analysing the Quality of Association Rules by Computing an Interestingness Measures. Indian Journal of Science and Technology 8 (15): 1-12, 2015.

Mari, D. And Kotz, S. Correlation and Dependence. Imperial College Press, London, 2001.

Phan, N. phdthesis. Ph.D. thesis, Université Monpellier 2, France, 2013.

PlancK, M. Instituto max planck de ornitologia. https:// www.orn.mpg.de/en, 2019.

Snowdon, C. T. O significado da pesquisa em Comportamento Animal. Estudos de Psicologia (Natal) 4 (2): 365-373, 1999.

TAYlor, R. Interpretation of the Correlation Coefficient: A Basic Review. Journal of Diagnostic Medical Sonography 6 (1): 35-39, 1990.

Torres, L. G., Orben, R. A., Tolkova, I., and Thompson, D. R. Classification of Animal Movement Behavior through Residence in Space and Time. PLOS ONE 12 (1): e0168513, 2017.

Vincenty, T. Direct and Inverse Solutions of Geodesics on the Ellipsoid with Application of Nested Equations. Survey Review 23 (176): 88-93, 1975.

Zhang, J., Oreilly, K. M., Perry, G. L. W., Taylor, G. A., And Dennis, T. E. Extending the Functionality of Behavioural Change-Point Analysis with k-Means Clustering: A Case Study with the Little Penguin (Eudyptula minor). PLoS ONE 10 (4): 1-14, 2015. 